\title{
一様磁場中における液体金属の透磁率と実効粘性の計測

\author{
Investigation of Magnetic Permeability and Effective-kinematic Viscosity of Liquid Metal \\ in Uniform Magnetic Field
}

\author{
○学 矢野可南子（北大院） 正 田坂裕司（北大） 正 村井祐一（北大） \\ 正 武田靖（北大） ル・ギャルパトリス（IRPHE） \\ Kanako YANO, Yuji TASAKA, Yuichi MURAI, Yasushi TAKEDA, \\ Hokkaido University, Kita-13, Nishi-8, Kita-ku, Sapporo \\ Patrice Le Gal, Institut de Recherche sur les Phénomènes Hors Equilibre, \\ 49 rue F. Joliot-Curie,BP146, 13384 Marseille, France
}

Key Words: MHD, Liquid metal, Magnetic permeability, Effective-kinematic viscosity

\section{Introduction}

MHD experiments in a large setup have been done to clarify the mechanism of the geo-dynamo. In order to simulate the phenomenon, huge Reynolds number and also huge magnetic Reynolds number should be realized in the setup. The magnetic Reynolds number is defined as

$$
R e_{\mathrm{m}}=\mu_{\mathrm{m}} \sigma U D,
$$

where $\mu_{\mathrm{m}}, \sigma, U$ and $D$ are respectively, magnetic permeability, electric conductivity, characteristic velocity and characteristic length of the setup. Magnetic diffusivity is also defined as

$$
\lambda=1 / \mu_{\mathrm{m}} \sigma,
$$

and thus the magnetic Reynolds number becomes

$$
R e_{\mathrm{m}}=U D / \lambda \text {. }
$$

Even a huge experimental setup with several meters in the characteristic length, its magnetic Reynolds number is incomparably smaller than that of the earth. The possibility to increase the magnetic Reynolds number is in decreasing magnetic diffusivity, e.g. by using liquid with high magnetic permeability. Usual liquid metals using for the MHD experiment are sodium ( $\mathrm{Na})$, mercury $(\mathrm{Hg})$ and gallium (Ga). The magnetic permeability becomes small by $\mathrm{Na}>$ $\mathrm{Hg}>\mathrm{Ga}$, however, order of these values are almost same. Moreover, using sodium, which has the largest magnetic permeability in these liquid metals, involves risk to react with water.

To solve this problem, we attempt to realize high magnetic permeability by using ferromagnetic particles. By mixing the ferromagnetic particle into the mercury, we may be able to increase the magnetic permeability dramatically. But we have to estimate this value to use it for the MHD experiment. In this study, we aim to establish the way to determine the magnetic permeability of unknown fluids by investigating spin-up of the fluid, and to verify its performance using well-known fluid. When the ferromagnetic fluid put into a uniform magnetic field, its kinematic viscosity may change. We also estimate the effective-kinematic viscosity of the ferromagnetic fluid under the uniform magnetic field.

\section{Experimental setup and experiment}

The illustration of the experimental setup is shown in Figure 1. The setup consists of a rotating open cylinder put in uniform magnetic field generated by a pair of Helmholtz coil, and measurement equipment. The cylinder is made of vinyl chloride and is rotated by a stepping motor via a belt. The inner diameter of the cylinder is 30 $\mathrm{mm}$. The inner diameter of coil is $260 \mathrm{~mm}$ and the outer is $275 \mathrm{~mm}$.
The distance of a pair of coils is $220 \mathrm{~mm}$. Rotating speed is determined by a rev counter mounted on the axis of the rotation. Testing fluid, Galinstan (GaInSn), is poured in the cylinder with covering by water to prevent oxidation. A probe for a gauss meter is mounted at outside of the rotating cylinder perpendicular to the direction of the magnetic field. The rotating cylinder and the Helmholtz coils are covered by a magnetic shield made of stainless steel to reduce noise.

When the cylinder starts rotation, advection of the fluid changes the orientation of the uniform magnetic field $B_{0}$. Different component of the magnetic field $\Delta B$ perpendicular to the uniform magnetic field appears and it depends on magnetic permeability $\mu_{\mathrm{m}}$, electric conductivity $\sigma$ and rotating speed $\Omega$, namely $\Delta B \sim \mu_{\mathrm{m}} \sigma B_{0} \Omega$. Magnetic diffusivity $\lambda=1 /\left(\mu_{\mathrm{m}} \sigma\right)$ can be determined by investigating the variation of the magnetic field for changing the rotation speed. The variation of the magnetic field detected by the gauss meter was recorded by an oscilloscope via an amplifier and a low-pass filter.

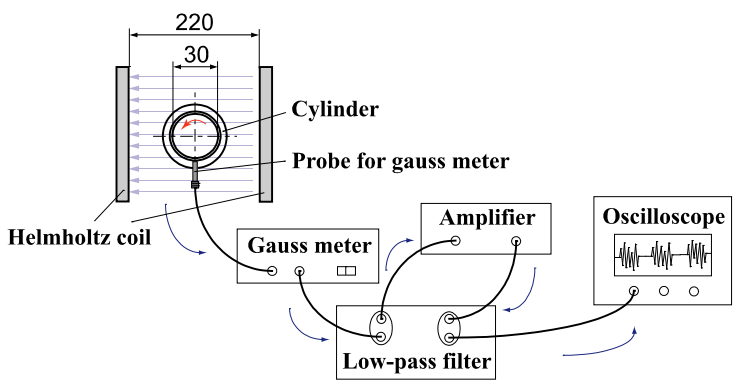

Fig.1 Experimental setup

\section{Results and discussions}

The magnetic field $\Delta B$, which is induced by advection of the fluid in the rotating cylinder, gradually increases with spin-up of the fluid, and it has the maximum value when the fluid is fully spin-up. Forward rotation and reverse rotation of the cylinder were repeated and the maximum value of $\Delta B$ is obtained as a mean value of the maximum values obtained at the each rotation; to reduce a long period fluctuation of the magnetic field. Figure 2 shows the maximum value at each rotating speed. The magnetic filed linearly increases with respect to the rotating speed.

We attempt to determinate magnetic permeability by using the slope of the relational expression between the magnetic field and the rotating speed explained in the last section. This slope is expressed 
as $B_{0} / \lambda, B_{0}$ is uniform magnetic field. In this study $B_{0}$ is $1.85 \mathrm{mG}$ so $\lambda$ is calculated as $0.389 \mathrm{~m}^{2} / \mathrm{s}$. The magnetic diffusivity of Galinstan is known as $0.361 \mathrm{~m}^{2} / \mathrm{s}$. The magnetic diffusivity calculated by the experiment agrees with the exact value, and magnetic permeability of Galinstan is determined as $0.80 \times 10^{-6} \mathrm{H} / \mathrm{m}$ for $\sigma=3.46 \times 10^{6}$ $\mathrm{S} / \mathrm{m}$.

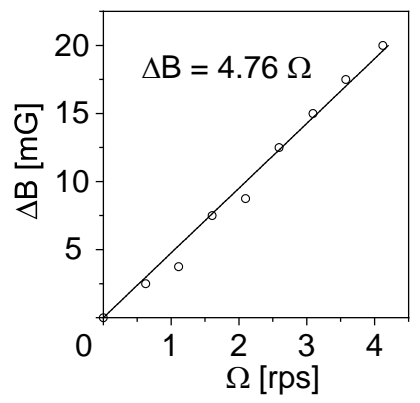

Fig.2 Maximum value of the perpendicular component of the magnetic field versus rotating speed

To calculate the spin-up time, $\tau$, from the obtained variation of the magnetic field, we use following equation to express the spin-up process;

$$
\Delta B(t)=\Delta B_{\max }\left[1-\exp \left(-\frac{t}{\tau}\right)\right] .
$$

This equation can be transformed into logarithmic expression,

$$
-\log \left(1-\frac{\Delta B(t)}{\Delta B_{\max }}\right)=\frac{t}{\tau},
$$

where $\Delta B_{\max }$ is the maximal value of the magnetic field. The left-side part of the equation is defined as $F(t)$. By fitting the obtained dataset, which is the temporal variation of the magnetic field just after the reverse rotation of the cylinder, to the equation (2), we obtains variations of $F(t)$ at each rotating speed as shown in Figure 3; the horizontal axis is measurement time and the vertical axis is $F(t)$. The black lines are linear approximated function. For the

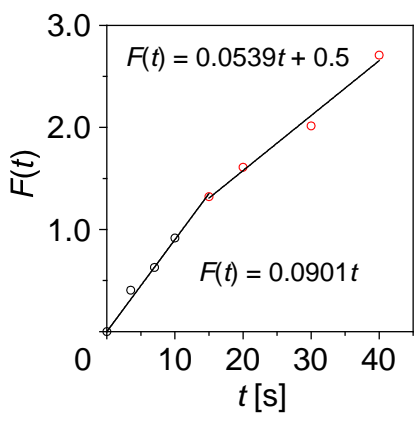

(a) $\Omega=66.7[\mathrm{rpm}]$

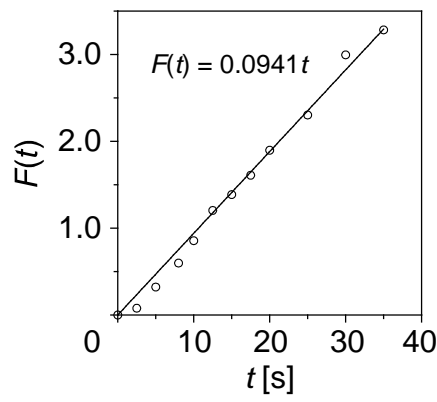

(b) $\Omega=247.3[\mathrm{rpm}]$

Fig.3 Dataset of the temporal variation of the magnetic field at each rotating speed and its fitting function lower rotating speed, two kinds of the slope exist. This is because turbulent flow due to the strong shear flow induced by reverse rotation of the cylinder and/or the momentum propagation from the bottom plate of the cylinder.

From the equation (2) $\tau$ is determined as the slope of $F(t)$. When two kinds of the slope exist, we selected the later one to calculate $\tau$. Double logarithmic plot of $\tau(\Omega)$ is shown in figure 4, where the black line represents the linear approximated function.

Spin-up time in the present configuration is determined by the following equation ${ }^{(1)(2)}$;

$$
\tau=\left(\frac{L^{2}}{v \Omega}\right)^{1 / 2} .
$$

In the logarithmic expression, it becomes

$$
\log (\tau)=-\frac{1}{2} \log (\Omega)+C,
$$

where $C$ is constant number. According to this expression, the slope of $\log (\Omega)$ is -0.5 and the slope of experiment result is almost the same value.

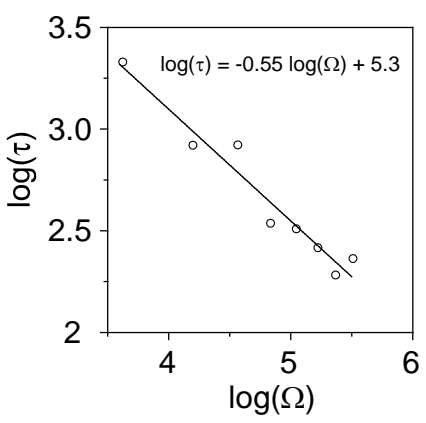

Fig.4 Spin-up time $\tau$ versus rotating speed $\Omega$ for Galinstan in the logarithmic expression

The effective-kinematic viscosity of Galinstan is calculated by utilizing modified equation (3),

$$
v=\frac{L^{2}}{\tau^{2} \Omega} .
$$

The effective-kinematic viscosity is determinate for each rotating speed, and the mean value of them becomes $2.21 \mathrm{~mm}^{2} / \mathrm{s}$. This value is twice larger than the actual value, around $1 \mathrm{~mm}^{2} / \mathrm{s}$. This difference may be caused by effects of Eckman layer and Stewartson layer, which are neglected in the equation (3).

\section{Conclusions}

We attempted to establish the method to measure the magnetic permeability and the effective-kinematic viscosity of unknown fluids by investigating spin-up of the fluid. Verification of the method has been done for Galinstan. Obtained results are reasonable and the applicability of the method has been confirmed. It is omitted here but the properties of mercury were also measured as reasonable values. Such other results will be presented at the conference. In the next step, we apply the established method for ferromagnetic fluid with changing amount of ferromagnetic particles.

\section{References}

(1) Greenspan, H. P. and Howard, L. N., J. Fluid Mech., 17, pp. 385-404, (1963)

(2) Benton, E. R. and Clark, A. Jr., Ann. Rev. Fluid Mech., 6, pp. 257-280, (1974) 